\title{
RTC-GWLAN: A Real Time Characterization of Generic IEEE 802.11 for High Density Services
}

\author{
Arinze C.O ${ }^{1}$, Idigo V.E ${ }^{2}$, Akpado K.A ${ }^{3}$, Agu V.N ${ }^{4}$, Nwalozie G. $C^{5}$ \\ 1, 2,3,5 Department of Electronic and Computer Engineering, Nnamdi Azikiwe University, Awka Nigeria \\ ${ }^{4}$ Department of Electrical Engineering, Nnamdi Azikiwe University, Awka Nigeria
}

\section{ABSTRACT}

This research investigates and analysed generic Wireless Local Area Networks (IEEE $802.11 \mathrm{a} / \mathrm{b} / \mathrm{g}$ ) in the context of their Quality of Service (QoS) in high density networks using some generic Key Performance Indicators (KPI) such as throughput (Bytes/Sec), Received Signal Strength Indicator (RSSI), and latency response (secs). The study focused on University of Nigeria Nsukka (UNN) which houses several hostels and faculties. Using Applied Research Methodology (ARM), this work characterized a real life generic hotspot testbed (UNN) for Received Signal Strength (RSS), mobile node distance, latency and throughput using Tranzio-Wavion Minipop Infrastructure in UNN as the network testbed. In this study, after sampling the selected KPIs, data were collected via our testbed measurements from the test nodes, Network Switching system/Network Operating Centres (NOCs) and their values were evaluated for an assessment study. The KPI results obtained showed that the traditional generic WLAN will scarcely scale in high density traffic environment in terms of scalability, speed performance (network convergence), cost, and other selected KPIs. This will lead to a proposal on a conceptual framework for Cognitive High Density Hotspot (CHDN) in our future work, which will address the limitations of generic WLANs for enterprise computing.

Keywords: Quality of Service (QoS); ARM; Key Performance Indicators (KPI); CHDN; WLAN.

\section{Council for Innovative Research}

Peer Review Research Publishing System

Journal: INTERNATIONAL JOURNAL OF COMPUTERS \& TECHNOLOGY

Vol 12, No. 6

editor@cirworld.com

www.cirworld.com, www.ijctonline.com 


\section{INTRODUCTION}

Generally, IEEE $802.11 \mathrm{a} / \mathrm{b} / \mathrm{g}$ WLANs are the most popular means of access to the Internet and intranet in clustered environments such as the Universities, Cybercafés, business centers, etc,. The proliferation of mobile devices equipped with WiFi interfaces, such as smart phones, laptops, and personal mobile multimedia devices, has heightened this trend of ubiquitous access. The performance of WiFi hotspots based on IEEE $802.11 \mathrm{a} / \mathrm{b} / \mathrm{g}$ serving locations such as large density environments and busy airports has been extremely poor as evidenced in user experience scenarios. In most cases for such settings, more than one WiFi access points (APs) (minipops) provide wireless access to the Internet for many user devices (STAs). However, the following are the commonly cited causes of this low scale performance as outline in [1]:

i. Contention and collision. When many STAs are competing for channel resources using CSMA/CA, the overhead of handling high contentions, such as carrier sensing, back-off and collisions, can be very high.

ii. Rate diversity and fairness. In an access network, different STAs may have different channel conditions. WiFi typically adopts an automatic rate adaptation scheme where poorer channel STAs use lower-rate modulation schemes which has a side effect of occupying longer channel time for transmission of the same size packets. As poorer STAs will use more channel resources, the overall throughput is reduced.

iii. Random losses and TCP Performance. Basically, TCP is the most commonly used transport protocol. But it treats all packet losses as congestion related and reduces its transmission rates for each packet loss. Unfortunately, wireless channels are prone to random packet losses unrelated to congestion. TCP throughput in WiFi can be low when many losses occur due to low channel quality and collisions.

iv. Traffic Asymmetry. In an access network like Basic Service Set (BSS), a single IEEE 802.11 AP_minipop can serve all associated clients. Moreover an analysis of network traces [2],[3] shows that downlink (AP to STAs) traffic is much greater than uplink traffic (4-10 times), attributable to commonly used client-and server based applications (e.g., web and email). The authors in [1] observes that this asymmetry of data traffic combined with IEEE 802.11 DCF providing equal opportunity for channel access to both APs and STAs results in congested APs and subsequent packet losses.

The outlined challenges have continued to affect the overall performance of generic WLANs based on IEEE 802.11. Sampled works in literature have failed to address poor quality of service in these networks (in the context of throughput, latency, RSSI, congestion management, channel fading, network scalability, etc) as their contributions suffers the following defects viz:

\section{WLAN Atomistic Performance Optimization}

The approaches sampled in [4],[5],[6],[7] to the performance optimization of WiFi are highly atomistic, focusing only on fixing one or a subset of the above articulated problems. In general, performance optimization must take a holistic approach with careful considerations of complex interactions among various control optimization metrics. Leveraging on wrong metrics for performance assessment KPIs will affect reasonable performance improvement in such networks, thereby negatively impacting the general performance.

\section{Deployment Challenges}

Existing solutions of generic WLANs for improved performance suffers from practical deployment because nearly all cases require changes in both APs/minipopos and clients and sometimes also in the MAC layer coordination that can be realized only by modifying the firmware of wireless interface cards.

While deploying a new (modified) AP is relatively easy in a hotspot, deploying client solutions for efficiency is difficult because of the diversity of client devices. For practical and incremental deployability, a proposed modification must be limited to AP.

\section{Lack of Real Test Workloads}

Existing IEEE 802.11 solutions in literature are not usually tested in real networks with realistic network workloads. Most of them are based on simulation or theoretical analysis [8],[9],[10]. There is a significant gap among the results predicted from real network experiments, simulation and theoretical analysis.

Consequently, it is hard to predict the actual performance of these proposed solutions for generic WLAN networks. We argue that all the enumerated factors could deter the deployment of generic WLAN solutions in production networks.

Since WiFi-based WLANs are widely used for Internet access, intranet, etc, the usage of a design model that allows the Access Points (AP) to serve few associated clients with symmetric uplink/downlink traffic patterns hotspots locations such as airports and large density environments will frequently experience poor performance in terms of downlink goodput and responsiveness in the context of QoS metrics. Based on the aforementioned limitations of the generic WLANs, Manzalini A. et al [11] proposes a self optimizing cognitive network of networks which uses decentralized cognitive plane (DCP) as a new separate plane to providing cross layer/node/network monitoring, knowledge acquisition and optimization in future networks. Alberto Rabbachin et al. [12] propose a cognitive network interference which uses the theory of truncated-stable distributions to develop a statistical model for cognitive networks (CNs) interference during spectrum access. While Nicola B. and Michele Z. [13] studied a Cognitive Access using Fuzzy Decision Making to propose a knowledge representation framework based on Fuzzy Logic which enables the implementation of a cognition process which is both cross-layer and 
network-aware. Thus, these few research efforts failed to integrate the effect of real life testbed in validation of their data analysis and evaluation which is more of theories and analysis.

In this paper, we demonstrate via empirical assessment studies, the measurement response in generic WLANs. We argue that solving the problem of traffic asymmetry will results in maximum performance improvements for large audience environments.

To validate our sample studies in literature while adding realism to our results, this paper will implement a solution assessment study in an off-the-shelf commercial IEEE $802.11 \mathrm{~g} \mathrm{AP}$, constructed a real network testbed of 10 IEEE 802.11 WiFi nodes and measured the performance of various optimization settings in network traffic loads emulating the traffic patterns captured from real traces of a switching system in a production testbed.

In this paper, we will outline the various limitations of generic WLAN following its performance degradation observations. In this regard, we concentrated and analyse a large network environment on our testbed with IEEE 802.11 nodes and the backend DataCenter servers. This traffic asymmetry results in maximum performance throughput, RSSI and latency such an environment will lead to our proposal on a Cognitive Hotspot Network (CHN) which (1) adaptively prioritizes AP's channel access over competing STAs avoiding traffic asymmetry (2) provides a fairness framework alleviating the problem of performance loss due to rate-diversity/fairness and (3) avoids degradation due to TCP behaviour. Future work will demonstrate that the proposed CHN not only improves downlink throughput by $400-700 \%$ but also reduces request's average response time (latency) by 30-40\%.

Hence, a validation model in the context of RSVP modulation, throughput, VLAN localization delay transition and service availability in the proposed $\mathrm{CHN}$ will show that the proposed cognitive hotspot network which shares similar ideology with the generic WLAN have better performance for high density hotspot environments.

This work in the subsequent sections will be organized as follows: Section 2 presents the research contributions for Large Density Audience Environments (LDAE). Section 3 discusses the research methodology, the study area, measurement processes and data collection strategy. In Section 4, we discuss the attributes of generic WLAN in the context of its performance evaluations based on our observations. In this section, the limitations of generic WLANs, the deductions from generic WLANs and a Proposal conceptual cognitive framework will be presented. In Section 5 , this work will carry out its discussion while concluding and making future recommendations in Section 6.

\section{RESEARCH CONTRIBUTIONS}

This paper concentrates on how to improve the performance of the IEEE $802.11 \mathrm{a} / \mathrm{b} / \mathrm{g} / \mathrm{n}$ networks by firstly understudying the generic WLAN using UNN as an LDAE. The key contribution of our work is to empirically study of the performance implications of adopting generic WLAN for high density traffic and services like multimedia services, cloud computing services, video, voice etc. Following the results of our findings, we will propose optimal solutions that will optimize the performance of G-WLAN which we tagged $\mathrm{CHN}$ for high density WiFi-hotspot services

\section{METHODOLOGY}

This paper will use an Applied Research Methodology (ARM) framework to systematically solve the research problem of generic WLANs. In this case, a quantitative strategy will be applied in the ARM. The benefit of ARM is that extensive study and characterization of a typical generic environment could be analyzed with the view of ascertaining its relative performance

index

Figure 1 shows our ARM framework. From figure 1, this work will first characterize a generic WLAN environment for high density hotspot 802.11 data access with a good test/experimental technique to derive our evidence which now gave rise to an improved approach to address performance issues of 802.11 WLAN hotspots. 


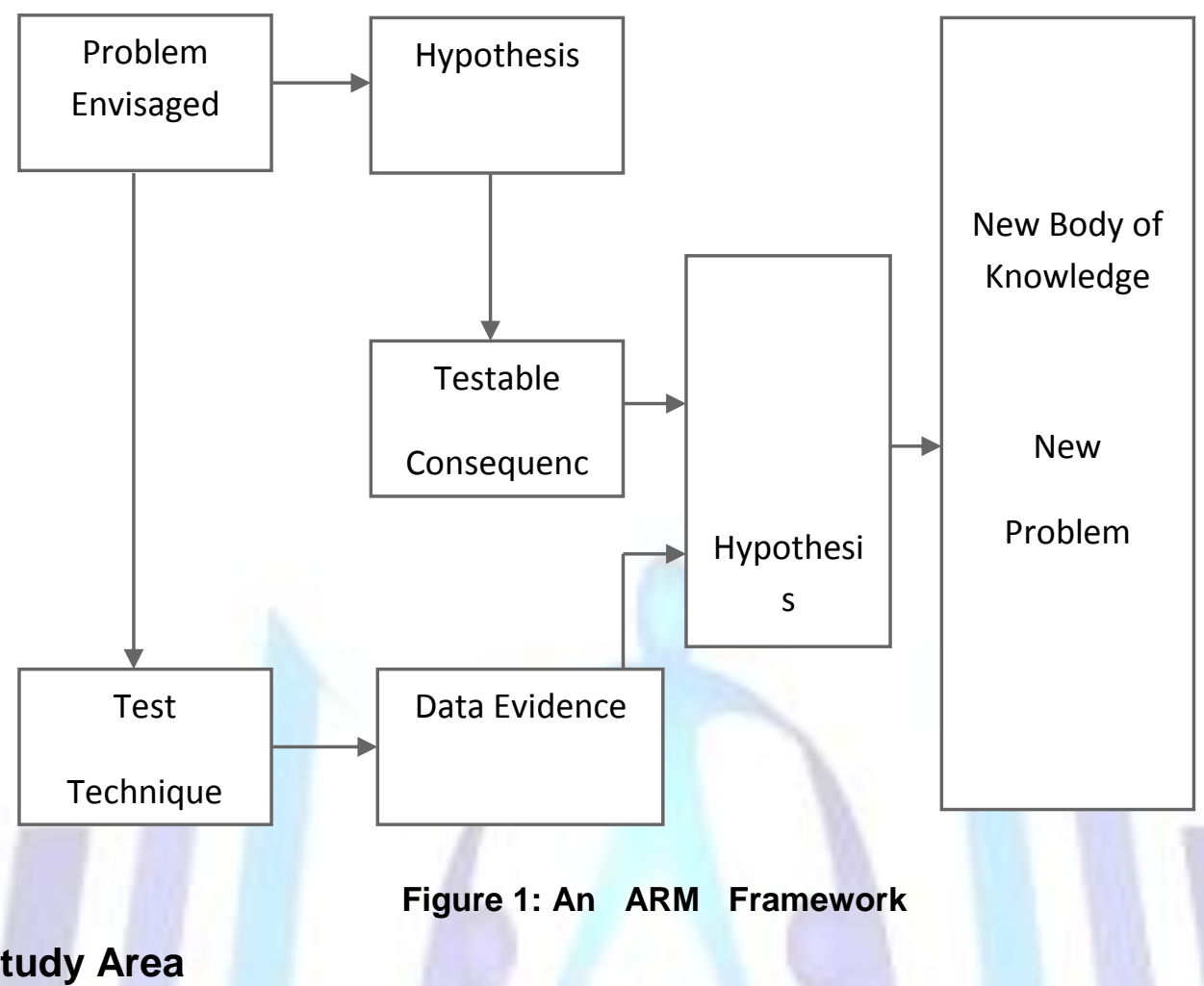

\subsection{Study Area}

The main campus of the University is located on 871 hectares of hilly savannah in the town of Nsukka, about eighty kilometres north of Enugu. The Nsukka campus houses the Faculties of Agriculture, Arts, Biological Sciences, Education, Engineering, Pharmaceutical Sciences, Physical Sciences, Social Sciences, and Veterinary Medicine while its Enugu campus has faculties of Business Administration, Environmental Studies, Law and Medical Sciences (see figure 2). The teaching hospital (UNTH) attached to the University is presently sited at Ituku-Ozalla (25 kilometres south of Enugu) on a 500 hectare site. And it also hosts the Faculty of Dentistry and Health Sciences and Technology.

Two hotspot environments drives network connectivity via the tranzeo TR-5800 Series (broadband wireless backhaul radios) and wavion hotspot technology. The wavion outdoor Wi-Fi base stations runs with $802.11 \mathrm{n}$ (WBSn) in $2.4 \mathrm{GHz}$ and $5 \mathrm{GHz}$ unlicensed bands and in $700 \mathrm{MHz}$ licensed band, offering end-to-end solutions including access, backhaul, CPEs, management and service provisioning tools. With up to nine embedded radios, $3 \times 3 \mathrm{MIMO}$ and three spatial data streams, Wavion's base stations can offers theoretical $450 \mathrm{Mbps}$ data speeds. The specification used for the access points are Wavion Base Station, $15806101,5.8 \mathrm{GHz}$ Spatially adaptive, multi-radio base station, with an array of 6 Omni antennas, 5.8GHz self backhaul, PoE input (PoE injector), Pole mount kit, FCC / TUV compliant.

An integration of the Wavion base station with the tranzeo backhaul radio is referred to as a Minipop (access point)

\subsection{UNN Campus (LDAE) - Measurement Environment}

We considered a large area of different locations as basic setting. Due to a potentially high number of users, seven minipops based on 802.11 APs that operate on different IEEE channels are placed within this area. Users appear in these locations at different points in time and at different places.

Firstly, looking at figure 3, we grouped seven different locations were seven respective Minipops are deployed namely viz: Ezeopi, MIS, Carver, Alvan, Abuja, ICT Danfodio, Nuga Minipops. Each minipop terminate in the data center switching system (see figure 2 and figure 3 ).

In this research, we used channel coding speed up to 5/6, OFDM symbol guard interval 400ns. For the OFDM symbol formation, we used 56 sub-carrier frequencies. Actually, with these specifications, the WLAN cards support only up to 72.2 Mbps data transmission. The result is the application-level good put, the real throughput, which user can get through data transmission. In our research effort, we are interested in the behaviour and performance of User Datagram Protocol (UDP) over wired and wireless networks with following setup: UDP Buffer Size: 41Kbytes, UDP Packet Size: 1500 Bytes.

Using ARM in figure 1, this work characterized a real life generic hotspot testbed for Received Signal Strength (RSS), Mobile Node distance, latency and throughput using Tranzio-Wavion Minipop Infrastructure in University of Nigeria Nsukka campuse as our generic testbed out of the University four campuses viz: - Nsukka (University of Nigeria, Nsukka, UNN), Enugu (University of Nigeria Enugu campus, UNEC), Ituku-Ozalla (University of Nigeria Teaching Hospital, UNTH) and Aba (University of Nigeria Aba campus, UNAC). Data collection was carried out in UNN environment only. 


\subsection{Tools of Measurement and Testbed}

In carrying out our measurements, these programs were used viz: Network stumbler and Iperf were installed on different laptop computers in the respective minipops while been monitored from a dedicated server running on mikrotik and ethereal wireshack. Network stumbler captures signal to noise ratio, latency, but throughput - used lperf program.

Considering the settings, to understand the basic performance of the IEEE802.11n during the test, we disabled Dynamic Host Configuration Protocol (DHCP), firewalls and other security settings. We used static stetting to avoid the additional resource consumption on the server, which is required to create a secure and flexible wireless connection. Our approach was to find the test place with good receptivity for various frame sizes, hence, the first experiment was conducted in the ICT centre (Nnmadi Azikwe library) as shown in figure 4. The location is an open building whose ground floor is used for the measurement. The distance of the building from the access point (minipop) is about $100 \mathrm{~m}$.

In this building, eight different VLAN scenarios were considered for point to point traffic on the minipops. The scenario includes

ICT_VLAN10,Ezeopi_VLAN20,MIS_VLAN30,Carver_VLAN40,Alvan_VLAN50,Abuja_VLAN60,Danfodio_VLAN70,and Nuga_VLAN80. For each VLAN Minipop, maximum allowable user is 1005 owing to the switch capacity.

From the ICT_VLAN minipop, we measured various network metrics from users (mobile hosts) scattering at different locations inside the building and to other minipops. Signal measurements were taken by following the network map of figure 2 and figure 3 .

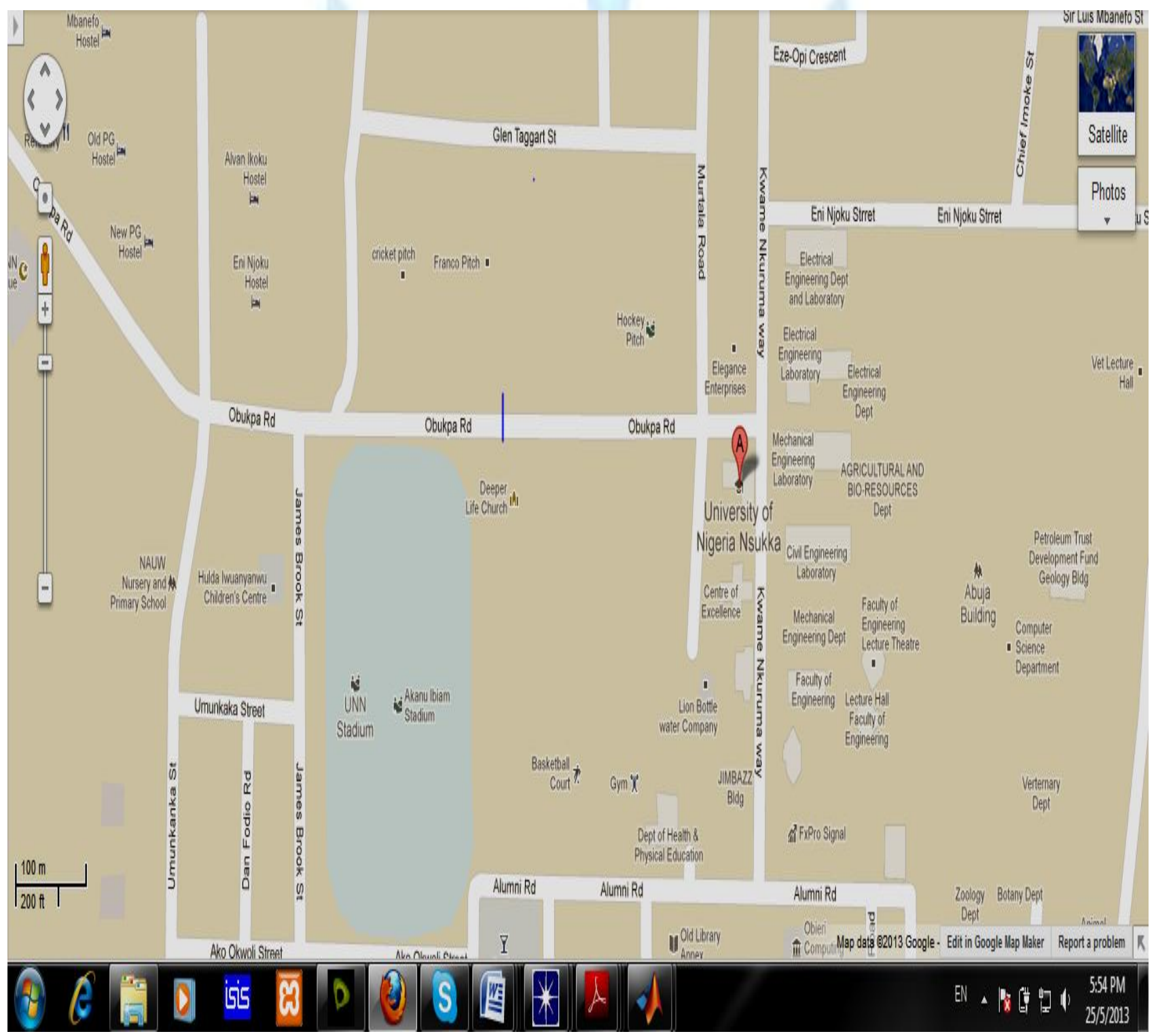

Figure 2: UNN Map Source

(https://maps.google.com.ng/maps?ie=UTF8\&q=university+of+nigeria\&fb=1\&gl=ng\&hq=university+of+ nigeria) 


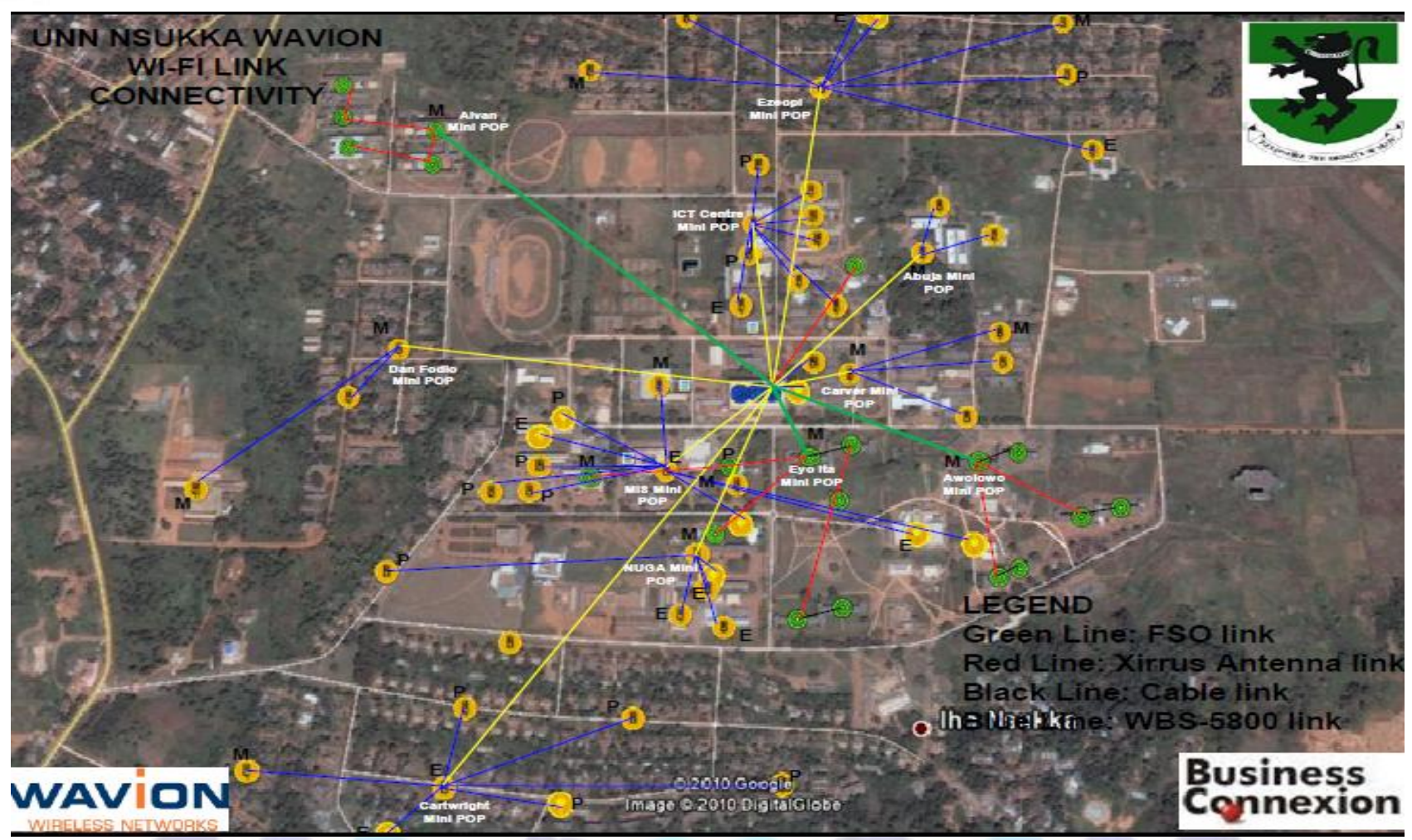

Figure 3: Network Map UNN generic 802.11 (Source UNN Wavion Wireless networks)

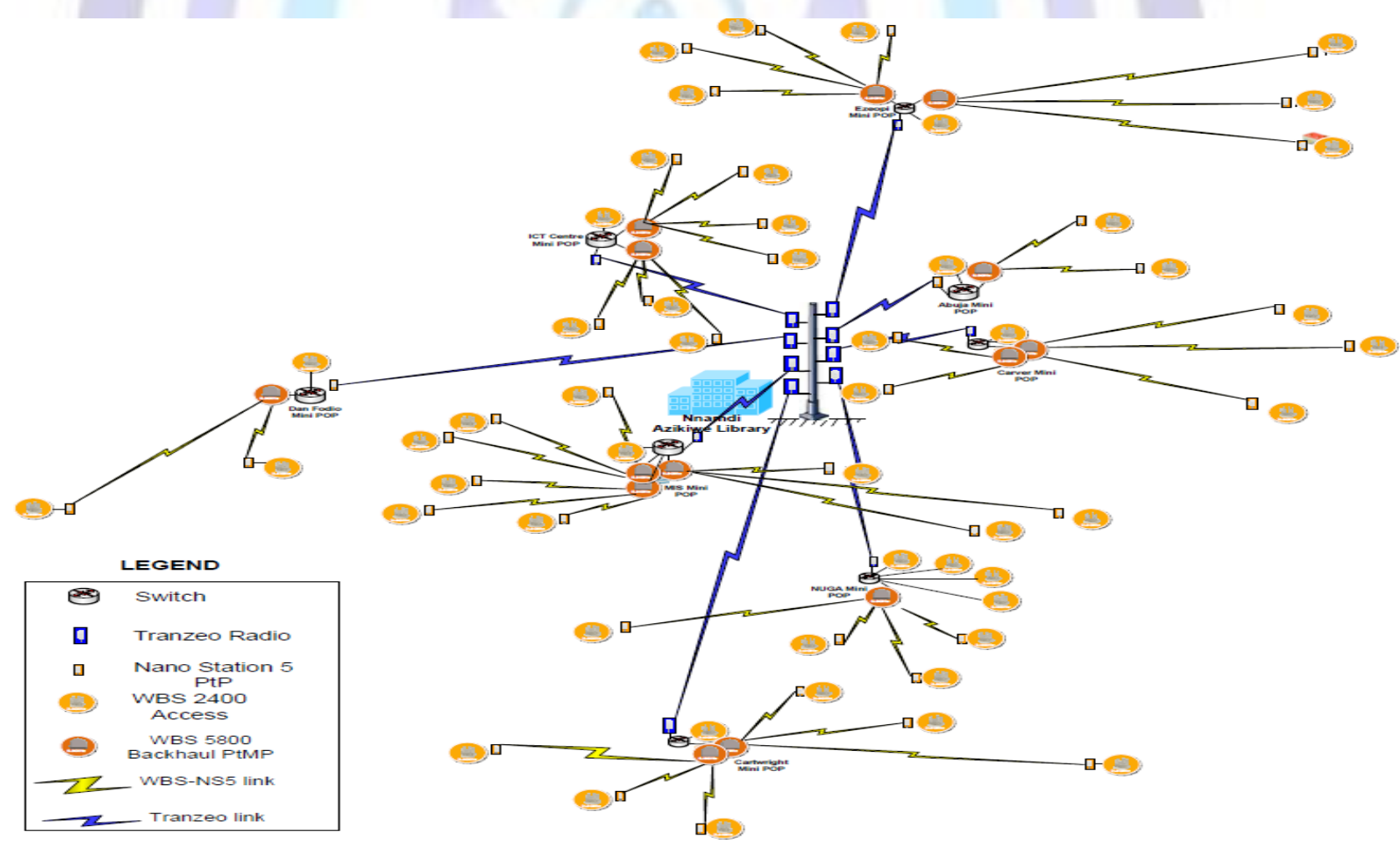

Figure 4: Design Diagram for Generic WLAN Testbed 


\subsection{Experimental Testbed and Measurement Procedure}

\subsubsection{Infrastructure Equipment Description}

To analyze the current wireless infrastructure considering the basic performance standards, we used the commercial off the Shelf (COTS) equipment with following configurations and settings viz:

\section{Switching DataCenter System}

For both testbeds, the minipops terminates at the DataCenter switching modules. The various VLANS were configured for user access. Figure 5 shows the UNN switching system in our setup.

\section{Minipop Tranzeo-Wavion Antenna}

In this work, the tranzeo TR-5800 Series which is a broadband wireless backhaul radios provide high data throughput over long distances. This was configured to drive data rates are up to $54 \mathrm{Mbps}$ at Half-Duplex speeds and $100 \mathrm{Mbps}$ at full duplex speed while operating in the license-free $5 \mathrm{GHz}$ frequencies. The TR-5800 Series was used for Point-to-Point wireless links (minipops) can also be used in Line-of-Sight (LoS) running at 5.3 to $5.8 \mathrm{GHz}$ backhaul point-to-point solution. The Wavion's 5800 beamforming technology is:

Based on Wavion's Beamforming Wi-Fi chip.

Leverages up to 6 radio and 6 high-gain antennas for optimal performance beamforming signal.

Performs per-packet true beamforming in both the uplink and downlink.

Exploits multi-path for its advantage by coherently combining all reflections, thus creating optimal signal at the receiver with up to $10 \mathrm{~dB}$ beamforming gain. This doubles the range, improves in building penetration, and increases aggregated base station capacity as air transmissions are more efficient.

- Inherently suppresses interferences by an average of $8 \mathrm{~dB}$, thus providing significant advantage in noisy environments.

Supports off-the-shelf standard Wi-Fi $802.11 \mathrm{a} / \mathrm{b} / \mathrm{g} / \mathrm{n}$ CPEs Wavion's base stations leverage on following unique technologies and capabilities which further enhance the technological lead.

Space Division Multiple Access (SDMA) technology - enables transmitting to multiple users simultaneously, thus further increasing the base station capacity.

- Dynamic Interference Handling (DIH) capability - enables continuous adaptation of the air access parameters according to the noise and interference level, thus maximizing the base stations capacity in noisy environments.

- Down-tilted antennas - further reduce the noise and interference levels.

- $\quad$ Automatic Channel Selection (ACS) capability - automatically selects the clearest radio channel for best radio performance.

Carrier grade - robust mechanical solution with bottom-up IP-67 design for reliable operation in extreme outdoor conditions, easy and fast installation, low power consumption, and enhanced management solution. Figure 6 shows the wavion nanostation used in the generic WLAN analysis.

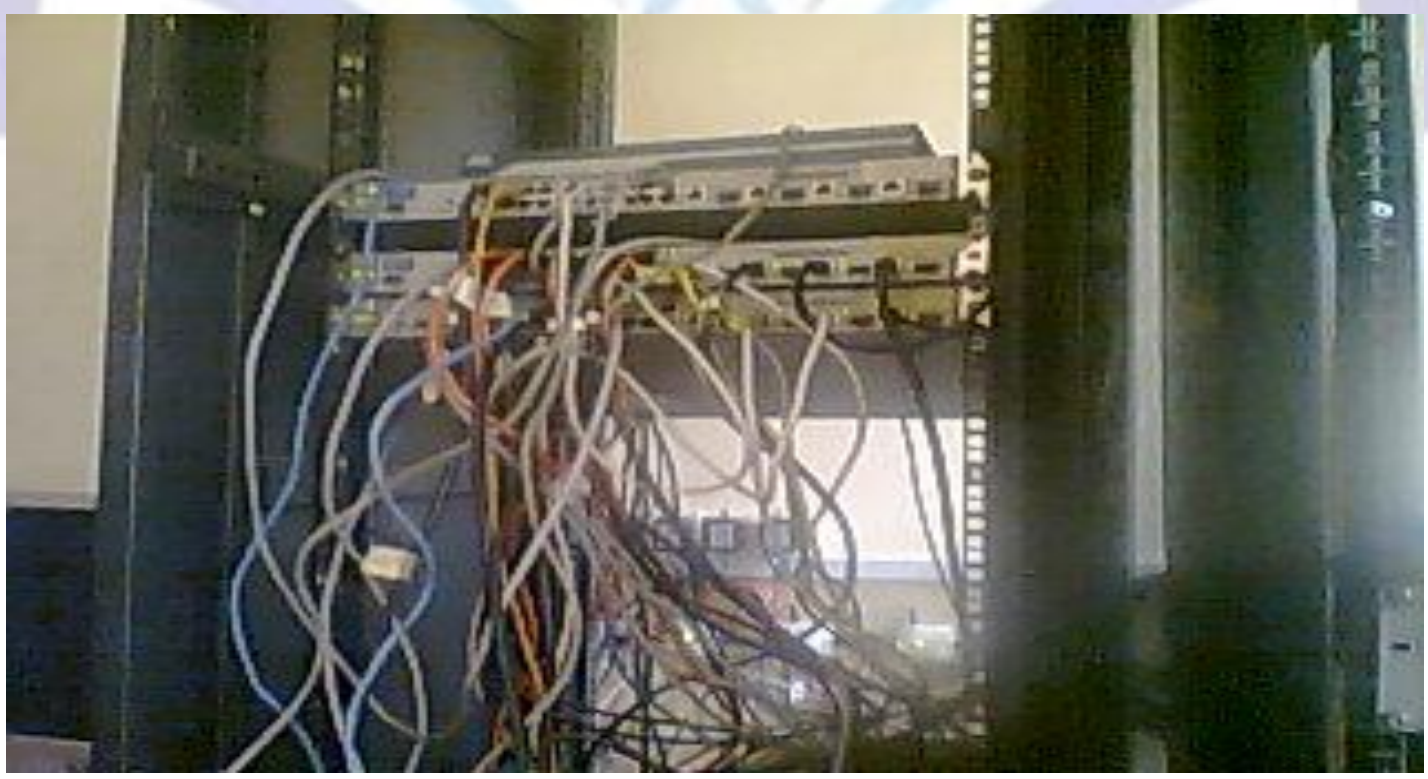

Figure 5: UNN Switching System (Sources: UNN ICT centre). 


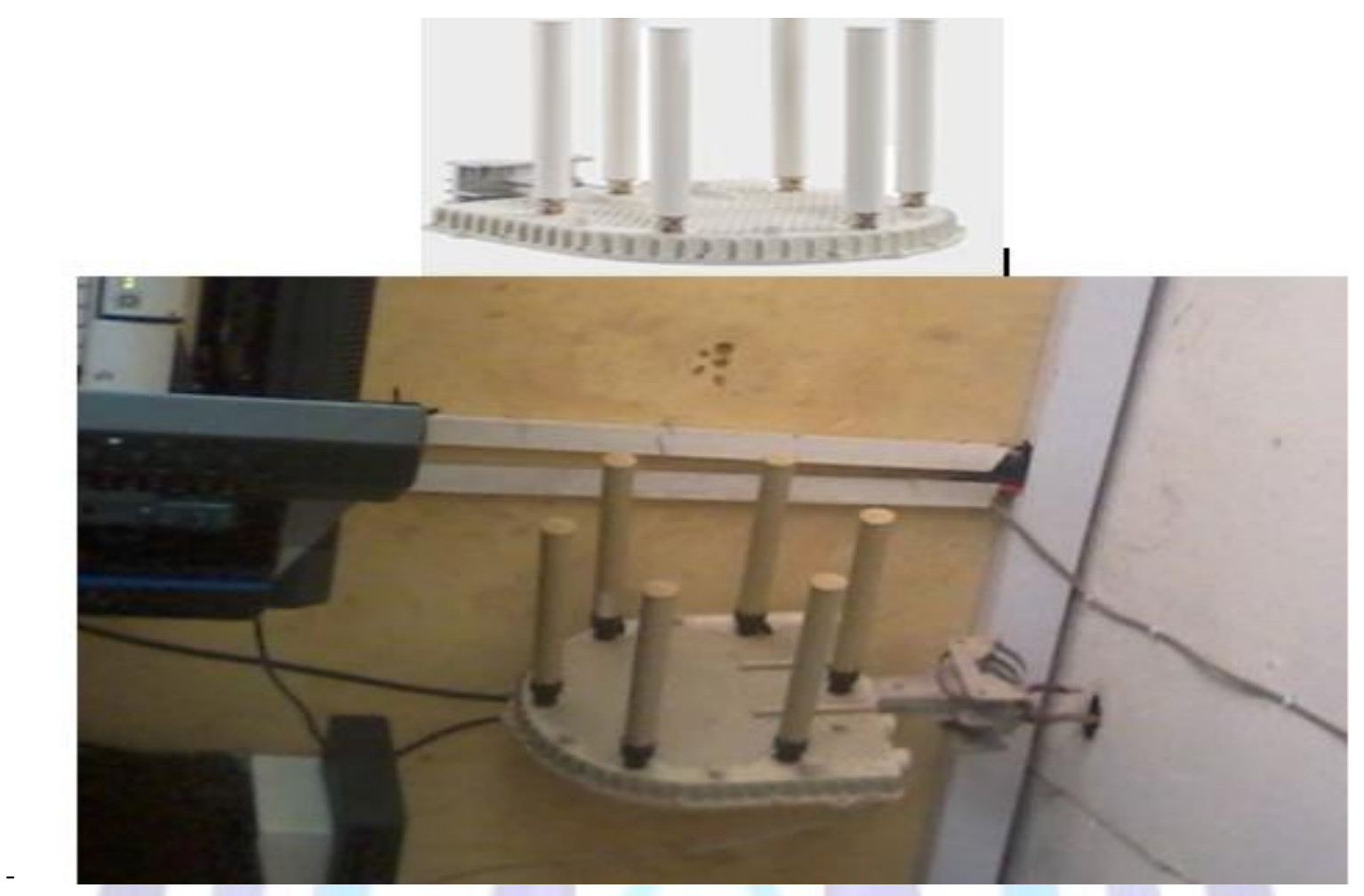

Figure 6: Wavion Nanostations (experimental testbed)

Figure 7 shows the Antenna mast with Tranzeo and wavion radios as captured by the researchers.

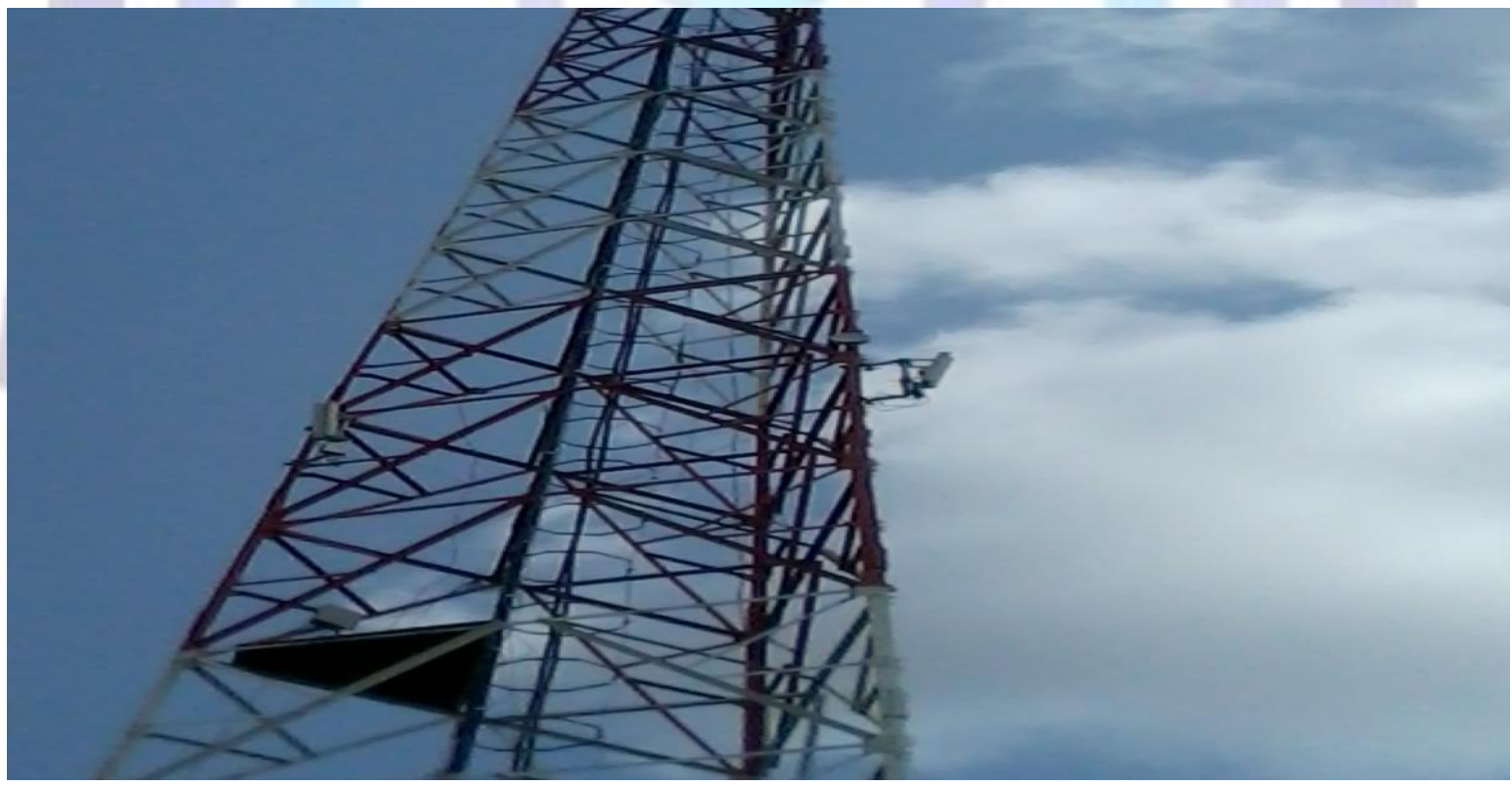

Figure 7: Antenna mast with Tranzeo and wavion radios (experimental testbed)

\subsubsection{Test System Configurations}

Computer 1 (IEEE802.11g):

Operating System: Windows Vista Home Premium (6.0, Build 6002);

Processor: AMD Turion (tm) 64×2 (2CPUs), 2,0GHz; 
Memory: 2046MB RAM;

Wireless g adapter: Atheros AR5007EG Wireless Network Adapter.

IEEE802.11g settings: additional channel coding speed up to 3/4, OFDM symbol guard interval $800 \mathrm{~ns}$ and $20 \mathrm{MHz}$ wide channel.

Computer 2 (IEEE802.11n):

Operating System: Windows 7 Home Premium 64-bit (6.1, Build 7601);

Processor: Intel(R) Core(TM) i3 CPU M380 @2.53GHz (4 CPUs), 2,5GHz;

Memory: 3072MB RAM;

Wireless $n$ adapter: The Broadcom BCM4329 802.11 network adapter.

IEEE802.11n limited settings without MIMO and 40MHz wide channel.

\subsection{Data Collection Design (Measurement in Outdoor)}

In this work, the switching DataCenter was configured to supports over 5000 user sessions with 2major servers (HP proliant 380G5, 370G5 server cluster model), 3800 switches (2) for 12 minipops, and two routers with SAN as a storage technique.

The server cluster, on MikroTik RouterOS V2.5 coordinates the traffic flow on the DCN. The coordinated interfaces are setup with following the TCP IP address viz: http://10.0.0.1 and http://10.0.0.5/Admin.

Users connect to network from their respective minipops from $8 \mathrm{am}-6 \mathrm{pm}$ in the DCN. The servers maintain reservations across the network and allocate tenant requests in an on-line fashion. Given our focus on quantifying the traffic performance in generic WLAN, this work then used Wireshark to capture network statistics alongside with the Microtic on the two-server IPs. In this work, over 1000 data captures were gathered for MIS_VLAN30, Carver_VLAN40,Alvan_VLAN50,Abuja_VLAN60,Danfodio_VLAN70, and Nuga_VLAN80, but we only focused on the point to point response of ICT_VLAN to Ezeopi_VLAN for our generic hotspot study. The parameters for data collection were captured in Table 1.

Table 1: Generic WLAN Parameters

\begin{tabular}{|l|l|}
\hline Generic WLAN Parameters & Specifications \\
\hline Link Connection & 8 Minipop POP \\
\hline Switch ports & 24 and 48 \\
\hline Terminal Test node Snifers & 2 major nodes \\
\hline Network Architecture & WLAN ESS \\
\hline Network Topology & Servers-In-Rack \\
\hline Server Clustering & NO \\
\hline Storage Area Network (SAN) & Yes \\
\hline VLAN & Yes \\
\hline RSVP & NO \\
\hline TCP & SACK \\
\hline Coverage & $500 m$ \\
\hline
\end{tabular}

Table 2: Measurement Dataset for Ezeopi_VLAN20

\begin{tabular}{|c|c|c|c|c|c|}
\hline $\begin{array}{l}\text { Number of } \\
\text { users }\end{array}$ & $\begin{array}{l}\text { Distances } \\
\text { (m) }\end{array}$ & $\begin{array}{l}\text { RSSI } \\
\text { (dB) }\end{array}$ & $\begin{array}{l}\text { Avg. Throughput } \\
\text { (bits/sec) }\end{array}$ & $\begin{array}{l}\text { Latency } \\
\text { (sec) }\end{array}$ & $\begin{array}{l}\text { Frame Sizes } \\
\text { (Hex-bytes) }\end{array}$ \\
\hline 10 & 5 & -68 & 79.6 & 0.012658228 & FFFFFFFFFFFFFFD0 \\
\hline 15 & 10 & -70 & 79.5 & 0.025316456 & FFFFFFFFFFFFFFD0 \\
\hline 20 & 15 & -80 & 79.4 & 0.037974684 & FFFFFFFFFFFFFFD1 \\
\hline 25 & 20 & -78 & 79.3 & 0.050632911 & FFFFFFFFFFFFFFD2 \\
\hline 30 & 25 & -72 & 79.2 & 0.063291139 & FFFFFFFFFFFFFFD0 \\
\hline
\end{tabular}




\section{PERFORMANCE EVALUATION}

As shown in figure 8, as the user density increases, the average throughput starts to drop from its peak threshold. This is peculiar with generic WLAN hotspots. Also, traffic does not flow across the core unless the switching system equipped with wireless support must control traffic between localized minipops and flows within the same RF neighbourhood, making users to experience less packet drops at bursty traffic periods. Bandwidth is a critical challenge, but the implication of the constrained bandwidth is that as the users uses high density services with low bandwidth allocation, the packet drop will be very significant.
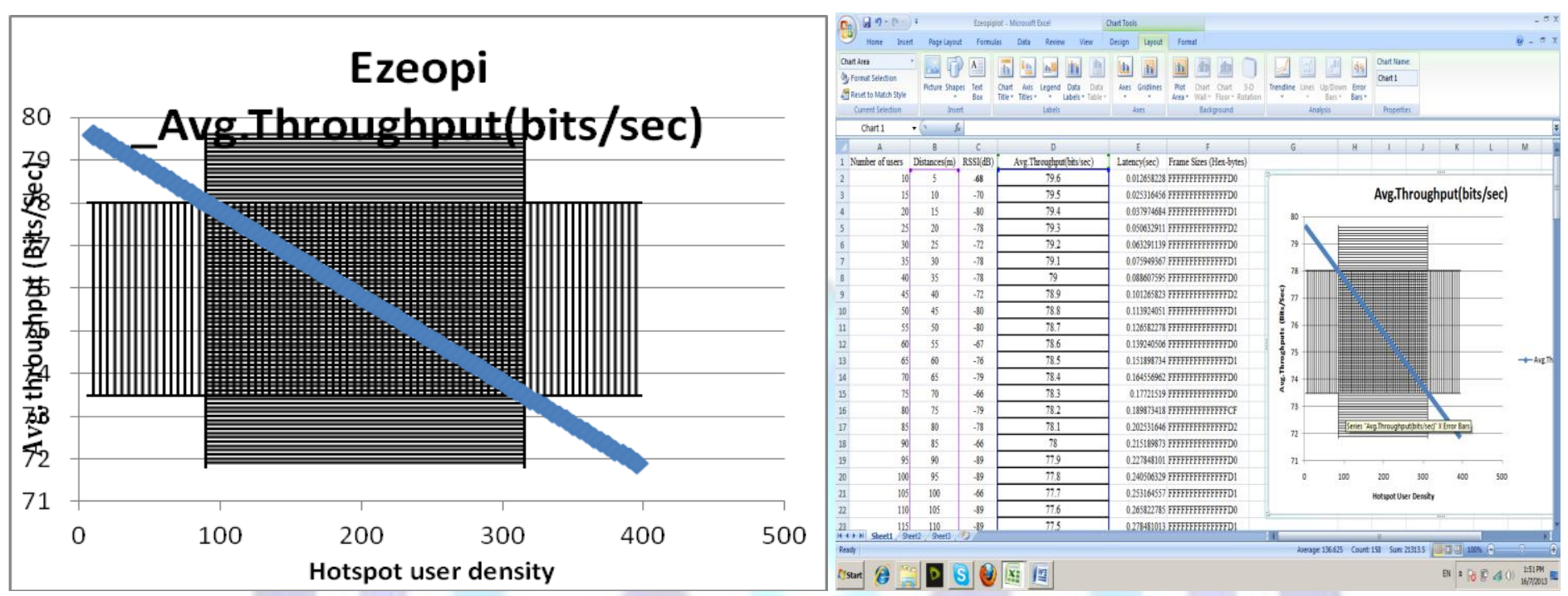

Figure 8: A Plot of Generic Throughput Behaviour

From figure 9, a thorough analysis of the Radio Signal Strength (RSS) model for distance estimation in UNN environment through an empirical quantification of user location metrics is shown. Received Signal Strength Indicator (RSSI) measures the strength of the radio signal received. Usually, calibration is required for mapping RSSI to distance values for the mobile node testers. We argue that RSSI does not necessarily imply or refer to the quality of the signal. But it is usually a 10 bit number, obtained from the physical layer, which could be used for tasks such as issuing of Clear-to-Send (CTS), etc. In the APs, the number of bits used for RSSI is hardware dependent as the radio on Minipop provides RSSI on the Analog to Digital Converter (ADC) channel 0 and is available in the lperf software running on the test machine as a 10 bit number. In the context of voltages, the following is the conversion from ADC Counts (10 bits) to RSSI in $\mathrm{dBm}$ as described in [14]:

$\mathrm{V}_{\text {RSSI }}=\mathrm{V}_{\text {batt }} \times$ ADC_Counts $/ 1024$

$R S S I_{(d B m)}=-51.3 \times V_{R S S I}-49.2$

Signal Strength (in $\mathrm{dBm})=x=10 \log _{10}(\operatorname{Power}(\mathrm{mW}) /(1 \mathrm{~mW}))$

(3)

In any case, the RSSI in dBm as shown in figure 8 is expected to be decreasing function with distance, but with our assumption on free space signal propagation, the response plot shows a wide range of unpredictability. This is expected because if we consider concentric spheres of increasing radius $r$ around the antenna, the total power radiated through the sphere remains constant, however depending on the minipop model, various technologies and schemes used in location estimation in hotspot environment could suffer from an assortment of errors and dispersions. The foundations for these deductions lie in the very nature of the RF medium. Noise and interference usually hampers the performance of the generic network because of the losses that they induce. These issues are relatively handled at the physical and MAC layers in the network protocol stack. But in case of distance measurement, noise and optical interference can lead to disastrous results by the induction of errors in the distance parameters measured by the devices. Therefore, there is always a need for accurate placement (calibration) of minipop APs for good location estimation judgements.

From figure 10, it is observed that the overall network delay gradually scales up with corresponding increase in the user density on the generic WLAN. We argue that this delay is unacceptable for high density hotspot domain as this will affect user experience adversely when using heavy TCP services like cloud services, video, etc. The plot of generic latency behaviour is an indicator of unscalable and unstable network in general. 

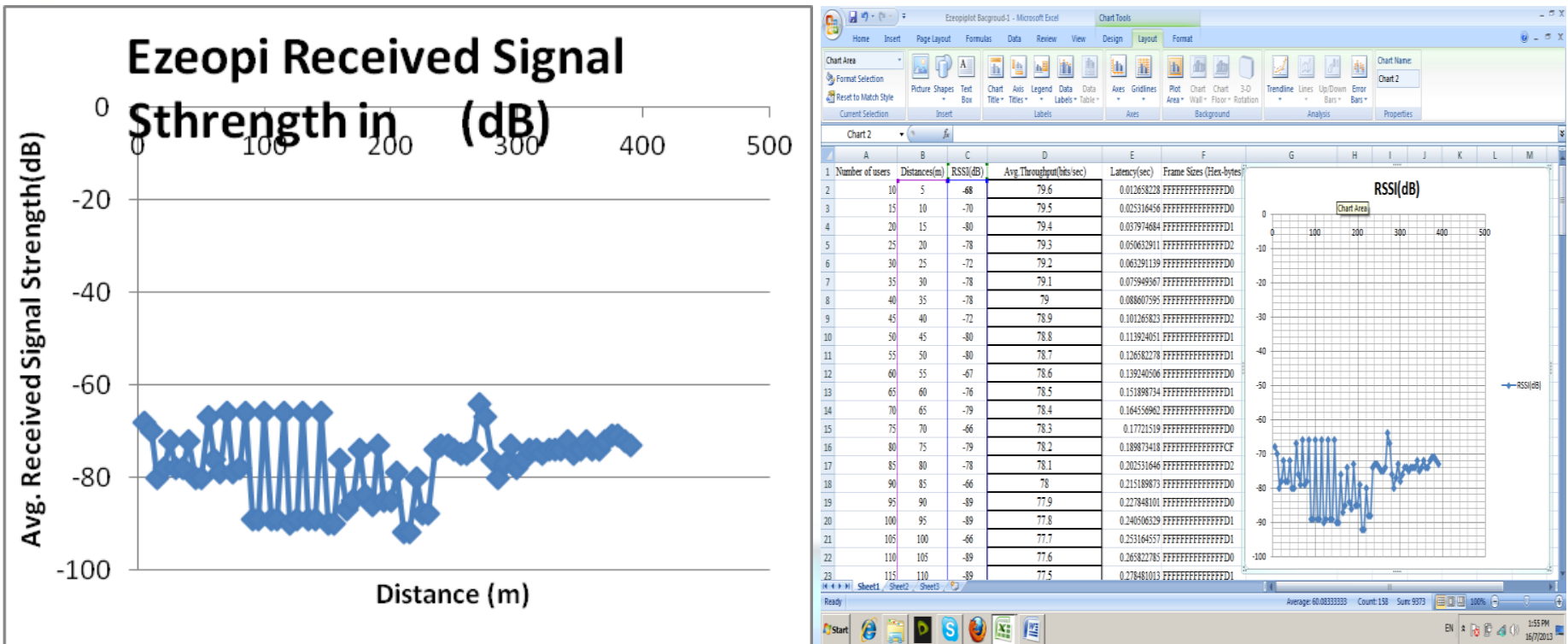

Figure 9: A Plot of Generic Signal Strength Behaviour

\section{Ezeopi Latency Response in (sec)}
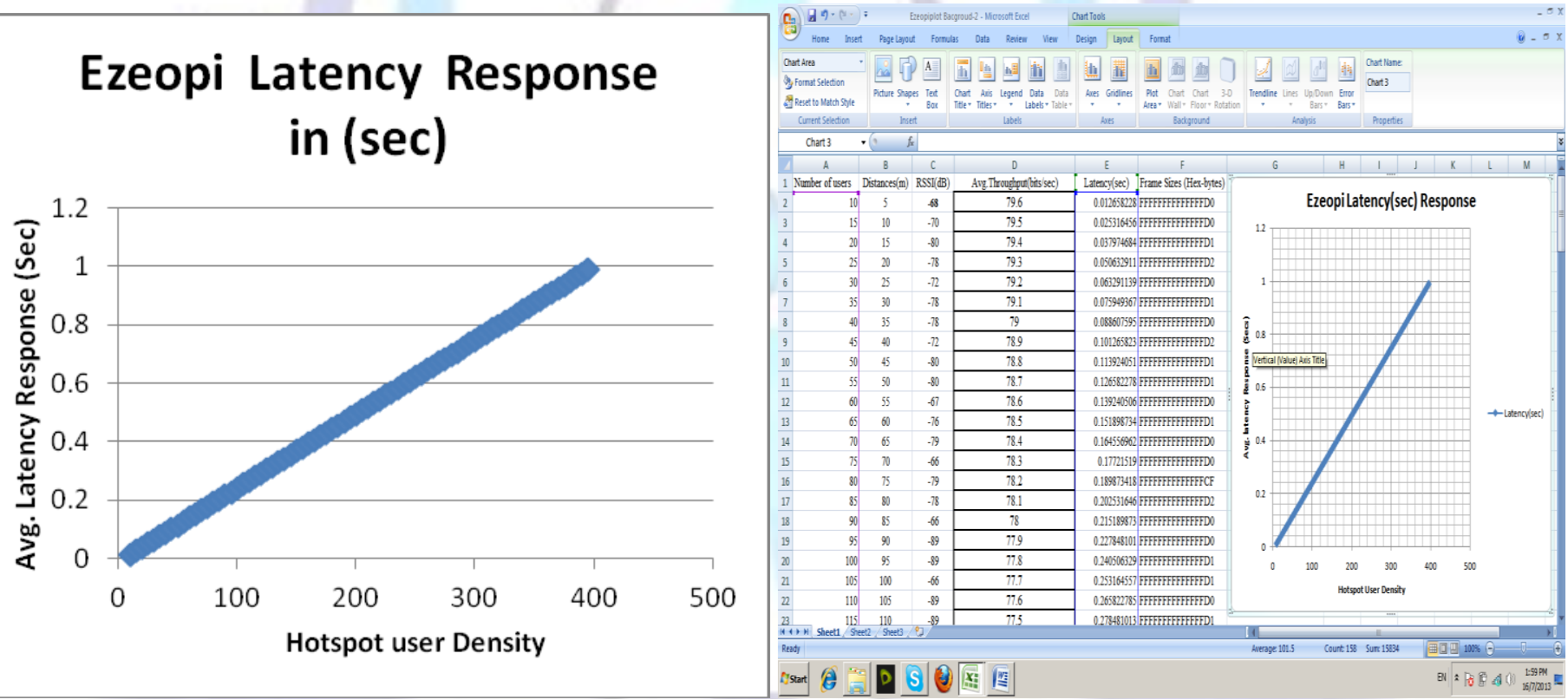

Figure 10: A Plot of Generic Latency Behaviour

\section{LIMITATIONS OF GENERIC WLAN BASED ON 802.11}

A coverage constraint across geographically dispersed minipops for data traffic is usually present. This now demands leverage on Extended Service Set Infrastructure resulting in enormous cost investment in generic hotspot WLANs.

1. In generic 802.11 WLAN hotspot, traffic does not flow across the core unless the switching system must control traffic between localized minipops and flows within the same RF neighbourhood, making users to experience packet drops at bursty traffic periods.

2. In generic 802.11 hotspot, data traffic is distributed across the network with bottlenecks into and out of a single device. Traffic destined to local resources like printers and workgroup traverses is highly vulnerable to the amount of bandwidth available and can also be vulnerable to attacks owing to weak cryptographic schemes.

3. Policy enforcement for network performance is dependent on the hotspot infrastructure set ups which lacks cognitive intelligence particularly at the access point core.

4. Poor Quality of service owing to high bit error rate at peak traffic periods with high network density.

5. The traditional TCP congestion control scheme is usually not optimized for high density hotspot scenario. 


\subsection{Deductions from traditional (Generic) Hotspot Testbed}

In this research, after our investigations and analysis, the following deductions were derived from our study viz:

\section{i. Scalability}

From the perspective of end users, the rapid growth of online/web services and applications that runs on hotspot 802.11 WLAN makes scalability a key design factor for our proposed cognitive WLAN design. A scalable hotspot network has the capacity for incremental expansion by adding more users, services and other infrastructure into the already operational structure without affecting the performance of the already existing running set up. This research observed that most hotspot setups are not scalable, hence, when the number of online user or web application of such network increases, the network will either be congested or crash completely.

\section{ii. Network Convergence and downtime}

The generic hotspot networks have problems of network convergence and downtime thereby disrupting services for an end user. One of the main concerns is for service continuity particularly via the AP minipops and the switching network. If a system becomes unavailable due to downtime, services may be impaired or stopped completely. It is necessary to provide a reliable infrastructure for avoidance of network downtime in order to minimize any chance of disruption.

\section{iii. Latency}

This is the amount of time that it takes for a packet to be transmitted from one minipop in the generic hotspot 802.11 network to another minipop in the network. In relation to the traditional hotspot model, the overall architecture generates over 30 percent network latency in switching and traffic delay, thereby negatively affecting responsiveness to end user service demands. This is because some of them were not based on technologies such as cognitive optimization framework which drastically reduces the number of network devices and service processes. Due to the bulky architecture of the generic hotspot networks, this results to about 30 percent network latency in switching. This of course affects the overall throughput negatively.

\section{iv. Throughput}

Throughput is a term used to describe the capacity of a system to transfer data. Since the demand for data exchange in generic hotspot environments is extremely enormous, a first design goal is to maximize the throughput at all cost. A good throughput will result to optimal performance.

Maximization of the throughput is equivalent to maximizing the link utilization. The amount of bandwidth allocated to different types of packets affect throughput considering the network user density.

\section{v. High Infrastructure Economy}

The cost of deployment as well as maintaining the generic hotspot IT infrastructure particularly for enterprise scalability is very immense and hence calls for a better approach to cost reduction as well as service availability. Since massive amounts of computational power is required to drive these hotspot systems, a better model for service provisioning with high quality of service at minimal cost will be widely accepted.

\section{vi. Network Congestion}

Owing to poor scalability in generic hotspot models, network congestion usually will degrade the performance of end user applications and services they run.

\section{DISCUSSION}

\section{Conceptual Cognitive Hotspot Network Framework}

Our investigations show that generic WLAN for high density networks lacks robustness and flexibility in the context of QoS metrics for high density network environment. Hence, in future work, the model of cognitive hotspot model reengineered for quality of service provisioning in a high density environment will address Congestion management using VLAN and Resource Reservation Protocol, Network scalability, and Adaptive Modulation Control for packet drop (hysteresis loss), and Co-interference Model for composite Minipops. Future work will derive formulations which the $\mathrm{CHN}$ model will use to address possible failures such as subnet break downs, connectivity issues while guaranteeing a multi-tenanted incremental upgrade on the generic WLAN scheme for high density hotspot environments.

Following our deductions earlier, for downtime avoidance and excellent QoS from users perspective (which will form our major drive for future work), we will show an intelligent (efficient) interconnection of Minipops for limitless subnets in high density hot spot environment that is capable of providing higher network capacity for efficient packet delivery and congestion free network. In improving the throughput, latency, RSS, etc, taking cognizance of the initialization processes of the generic WLAN infrastructures, such as the Minipop switch interconnection in the CHN architecture, we, shall introduce the following in our reengineering in the future work viz:

- An Analytical model that will handle traffic control issues in between the switching system and the mobile user end device 
- An Adaptive Modulation Control Scheme for Hysteresis and low SNR which is meant to suppress or normalize fading effect while being fault-tolerant at the same time.

- Subnet location isolation for every user in the cognitive hotspot architecture.

- An MLS reengineering for Virtual LAN (VLAN) and Resource Reservation Protocol (RSVP) for broadcast traffic optimization.

Consequently, in designing our proposed cognitive hotspot model, the main goals are to maintain throughput, low latency, fast convergence and scalable network that are flexible with minimal downtime and less administrative overhead.

The network should be capable of supporting high performance workloads (HPW) such as heavy web applications, data and video services. Meanwhile, multiple flows from various subnets should be localized within such subnets, but still share link capacity of the MLS fairly. The performance of the network will be measured by QoS parameters such as throughput, latency, and service availability from the end user application perspective.

\section{CONCLUSION AND FUTURE WORK}

This work opines that providing convenient QoS mobile users in high density network environments has attracted critical attention since wireless LANs are deployed in academies and enterprises. Since many related projects rely on centralized resource management in their mobile access design, these networks uses at least one DHCP server, Kerberos authentication and ticket granting servers, RADIUS server, and SNMP server for resource management at the time of mobile access. A significant difference between decentralized CHN and these centralized BSS schemes is service availability via good QoS. Consequently, in this paper, we have carried out a comprehensive assessment study on a real life testbed with an in-depth characterization using UNN as a case study. The generic WLAN is shown to have limitations and as such cannot scale well in high density network scenarios. Our proposed $\mathrm{CHN}$ is a decentralized location independent system based on well structured integrations which makes for easy-of deployment-and-use, efficiency in QoS as well as offering a well defined secured system that connects mobile guests to secured wireless switching center. The $\mathrm{CHN}$ box ensures all three aspects of mobile privacy, namely content privacy, identity privacy, and location privacy is assured. The implementation cost of $\mathrm{CHN}$ is very low and the result system envisaged being efficient and potentially mobile. So far we are still evaluating the proposed CHN content privacy services in a real testbed. In the near future, we will validate our $\mathrm{CHN}$ proposal using a good simulation tool.

\section{ACKNOWLEDGEMENT}

The Authors wish to thank the ICT Centre of University of Nigeria Nsukka for assisting and providing the required facilities to conduct this study

\section{REFERENCES}

[1]. Arpit Gupta, Jeongki Min, Injong Rhee,"WiFox: Scaling WiFi Performance for Large Audience Environments", CoNEXT, Nice, France Pp. 10-13, 12 December 2012.

[2]. M. Rodrig, C. Reis, R. Mahajan, D. Wetherall, and J. Zahorjan."Measurement. Based characteristics of 802.11 in a Hotspot setting. In ACM E-WIND, 2005.

[3]. A. Schulman, D. Levin, and N. Spring. "On the fidelity of 802.11 Packet Traces." In PAM, 2008.

[4]. E. Aguilera, J. Casademont, J. Cotrina, and A. Rojas. Perf., "Enhancement of WLAN IEEE 802.11 for asymmetric traffic".In IEEE PIMRC, 2005.

[5]. X. Wang and S. A. Mujtaba. "Performance Enhancement of 802.11WLAN for asymmetry traffic using an Adaptive MAC layer Protocol", In IEEE VTC, 2002.

[6]. J. Jeong, S. Choi, and C. Kim. Achieving weighted fairness between Uplink and downlink in IEEE 802.11 DCF-based WLANs". In QShine, 2005.

[7]. S. Shin and H. Schulzrinne. "Balancing uplink and downlink delay of VolP traffic in WLANs using Adaptive Priority Control (APC)". In ACM QShine, 2006.

[8]. R. Bruno, M. Conti, and E. Gregori. "Design of an enhanced AP to optimize TCP perf. in Wi-Fi hotspot networks". Wirel.Netw., 2007.

[9] F. Keceli, I. Inan, and E. Ayanoglu."Weighted Fair Uplink/Downlink Access Provisioning in IEEE 802.11 eWLANs". In IEEE ICC, 2008.

[10] S. W. Kim, B. Kim, and Y. Fang. "Downlink and Uplink Resource Alloc", in IEEE 802.11 wlans. IEEE TVT, 2005. 
[11]. A. Manzalini, P.H. Deussen, S. Nechifor, M. Mamei, R. Minerva1,C. Moiso1, A. Salden, T. Wauters And F. Zambonelli, "Self-optimized Cognitive Network of Networks",The Computer Journal Advance Access publisher, March 23, 2010.

[12]. Alberto Rabbachin, Tony Q.S. Quek, Hyundong Shin and Moe Z. Win, "Cognitive Network Interference", IEEE Journal On Selected Areas In Communications, Vol. 29, No. 2, pp 480-498, February 2011.

[13]. Nicola Baldo and M Zorzi, "Cognitive network access using fuzzy decision making", IEEE International Conference, Vol 35, pp 6504-6510, June 2007.

[14]. Chipcon. ChipCon CC1000 R/F Module Datasheet. ChipCon Datasheets, Oslo, Norway, 2002 